VERSITA

Biometrical Letters

DOI:10.2478/bile-2013-0006

Vol. 49 (2012), No. 2, 81-94

\title{
The extreme value Birnbaum-Saunders model, its moments and an application in biometry
}

\author{
M. Ivette Gomes ${ }^{1}$, Marta Ferreira ${ }^{2}$, Víctor Leiva ${ }^{3}$ \\ ${ }^{1}$ Universidade de Lisboa, DEIO, CEAUL and FCUL, Portugal, \\ e-mail: ivette.gomes@fc.ul.pt \\ ${ }^{2}$ Departamento de Matemática, Universidade do Minho, Portugal \\ ${ }^{3}$ Departamento de Estadística, Universidad de Valparaíso, Chile
}

\section{SUMMARY}

The Birnbaum-Saunders (BS) model is a life distribution that has been widely studied and applied. Recently, a new version of the BS distribution based on extreme value theory has been introduced, named the extreme value Birnbaum-Saunders (EVBS) distribution. In this article we provide some further details on the EVBS models that can be useful as a supplement to the existing results. We use these models to analyse real survival time data for patients treated with alkylating agents for multiple myeloma. This analysis allow us to show the adequacy of these new statistical distributions and identify them as models useful for medical practitioners in order to predict survival times for such patients and evaluate changes in their treatment dose.

Key words: extreme value theory, parametric modelling, survival data

\section{Introduction and preliminaries}

The Birnbaum-Saunders (BS) model is a life distribution that was introduced and studied by Birnbaum and Saunders (1969). This distribution has been widely applied in recent decades. BS and standard normal random variables (RVs), now denoted respectively by $T$ and $Z$, are related by the formula

$$
T=\delta\left(\alpha Z / 2+\sqrt{\{\alpha Z / 2\}^{2}+1}\right)^{2} \quad \text { i.e., } \quad Z=(\sqrt{T / \delta}-\sqrt{\delta / T}) / \alpha,
$$

with $\alpha>0$ and $\delta>0$ being shape and scale parameters. Thus when a RV $T$ follows a BS distribution with parameters $\alpha$ and $\delta$, the notation $T \sim \operatorname{BS}(\alpha, \delta)$ is used. In addition, let us consider the usual notations $\phi$ 
and $\Phi$ for the standard normal probability density function (PDF) and cumulative distribution function $(\mathrm{CDF})$, respectively, and let

$a_{t}=(\sqrt{t / \delta}-\sqrt{\delta / t}) / \alpha$, so that $a_{t}^{\prime}=d a_{t} / d t=(\sqrt{t / \delta}+\sqrt{\delta / t}) /(2 \alpha t)$.

Then, the PDF and the CDF of $T$ are respectively

$$
f_{T}(t)=\phi\left(a_{t}\right) a_{t}^{\prime} \quad \text { and } \quad F_{T}(t)=\Phi\left(a_{t}\right), t>0,
$$

with $a_{t}$ and $a_{t}^{\prime}$ defined in (1).

The assumption of a normal RV $Z$ can obviously be relaxed, supposing that it follows any other distribution with $\operatorname{PDF} f_{Z}$. We then obtain a general BS type (BST) RV, denoted by

$$
T \sim \operatorname{BST}\left(\alpha, \delta ; f_{Z}\right), \quad \text { with a } \operatorname{PDF} \quad f_{T}(t)=a_{t}^{\prime} f_{Z}\left(a_{t}\right), t>0,
$$

again with $a_{t}$ and $a_{t}^{\prime}$ given in (1). Among those models, we mention the extreme value Birnbaum-Saunders (EVBS) distributions, recently introduced by Ferreira et al. (2012), essentially based on results from extreme value theory (EVT).

In Section 2 of this article we present a few results on EVT. In Section 3 we introduce the EVBS models, providing information on their moments. In Section 4 we discuss estimation and model checking for this type of models. In Section 5 we make some comments on the importance of hazard analysis. In Section 6 we provide an application to biometrical data. Finally, in Section 7, we provide some concluding remarks.

\section{Limiting results in EVT}

The main limiting result in EVT dates back to the papers by Fréchet (1927), Fisher and Tippett (1928), von Mises (1936) and Gnedenko (1943). These authors fully characterized the possible non-degenerate limit laws of the sequence of maximum values, $X_{n: n}$, suitably normalized, as $n \rightarrow \infty$, proving what is called Gnedenko's extremal types theorem. More specifically, all possible non-degenerate weak limit distributions of the normalized partial maxima, $X_{n: n}$, of independent and identically distributed (IID) RVs, $X_{1}, \ldots, X_{n}$, are generalized extreme value $(\mathrm{GEV})$ distributions. That is, if there are normalizing constants $a_{n}>0, b_{n} \in \mathbb{R}$ and some non-degenerate CDF, $G$, such that, for all $x \in \mathcal{C}(G)$, the set of continuity points of $G$,

$$
\lim _{n \rightarrow \infty} \mathrm{P}\left\{\frac{X_{n: n}-b_{n}}{a_{n}} \leq x\right\}=G(x),
$$


we can redefine the constants in such a way that

$$
G(x) \equiv G_{\gamma}(x):= \begin{cases}\exp \left(-(1+\gamma x)^{-1 / \gamma}\right), 1+\gamma x>0, & \text { if } \gamma \neq 0 \\ \exp (-\exp (-x)), x \in \mathbb{R}, & \text { if } \gamma=0,\end{cases}
$$

given in the von Mises-Jenkinson form (see von Mises, 1936; Jenkinson, 1955) and denoted as $\operatorname{EV}_{\mathrm{M}} \equiv \mathrm{EV}_{\mathrm{M}}(\gamma)$ laws. We then say that the CDF $F$ underlying the RVs $X_{1}, X_{2}, \ldots$, is in the max-domain of attraction (MDA) of $G_{\gamma}$, in (4), and use the notation $F \in \mathcal{D}_{\mathrm{M}}\left(G_{\gamma}\right)$. The limiting CDFs, $G$, in (3), are then max-stable (MS), i.e., they are indeed the unique laws $S$ such that the functional equation $S^{n}\left(\alpha_{n} x+\delta_{n}\right)=S(x)$, for $n \geq 1$, holds for some $\alpha_{n}>0$ and $\delta_{n} \in \mathbb{R}$. The real parameter $\gamma$ in (4), corresponding to the primary parameter of interest in EVT, is the so-called extreme value index. This index governs the behaviour of the right-tail of $F$. The GEV distribution, a unified version of all possible non-degenerate weak limits of maxima of sufficiently long sequences of IID, or more generally weakly dependent and stationary RVs, reduces to the Fréchet $(\gamma>0)$, max-Weibull $(\gamma<0)$ and Gumbel $(\gamma=0)$ CDFs respectively. In fact, the GEV distribution in (4) is often separated into the three following types:

$$
\begin{array}{ll}
\text { Type I (Gumbel) : } & \Lambda(x)=\exp (-\exp (-x)), x \in \mathbb{R}, \\
\text { Type II (Fréchet) : } & \Phi_{\alpha}(x)=\exp \left(-x^{-\alpha}\right), x \geq 0, \\
\text { Type III (max-Weibull) }: & \Psi_{\alpha}(x)=\exp \left(-(-x)^{\alpha}\right), x \leq 0,
\end{array}
$$

with $\gamma=0, \gamma=1 / \alpha>0$ and $\gamma=-1 / \alpha<0$, respectively. We have $\Lambda(x)=G_{0}(x), \Phi_{\alpha}(x)=G_{1 / \alpha}(\alpha(1-x))$ and $\Psi_{\alpha}(x)=G_{-1 / \alpha}(\alpha(x+1))$, with $G_{\gamma}$ being the GEV distribution given in (4). For a recent overview of similar topics in the field of EVT, see Gomes et al. (2008).

Remark 1. All results developed for maxima can easily be reformulated for minima since $X_{1: n}:=\min \left\{X_{1}, \ldots, X_{n}\right\}=-\max \left\{-X_{1}, \ldots,-X_{n}\right\}$. If we are interested in the left-tails, i.e. in the limiting behaviour of the sequence of minimum values, we have for a linearly normalized minimum, a limiting $\mathrm{CDF}, G_{\gamma}^{*}(x)=1-G_{\gamma}(-x)$, with $G_{\gamma}(\cdot)$ given in (4), often referred to as an $\mathrm{EV}_{\mathrm{m}} \equiv \mathrm{EV}_{\mathrm{m}}(\gamma)$ law, i.e.

$$
G_{\gamma}^{*}(x)= \begin{cases}1-\exp \left(-(1-\gamma x)^{-1 / \gamma}\right), 1-\gamma x>0, & \text { if } \gamma \neq 0, \\ 1-\exp (-\exp (x)), x \in \mathbb{R}, & \text { if } \gamma=0 .\end{cases}
$$


We then say that $F$ belongs to the min-domain of attraction of $G_{\gamma}^{*}$, in short $F \in \mathcal{D}_{\mathrm{m}}\left(G_{\gamma}^{*}\right)$. The parameter $\gamma$, in (5), determines the left-tail behaviour of $F$, as the parameter $\gamma$, in (4), determines the right-tail behaviour of $F$, both thus being crucial parameters in EVT.

In Figure 1, we represent the right-tails of truncated positive $\mathrm{EV}_{\mathrm{M}}$ and normal PDFs, as well the BS(1,1) PDF. If $\gamma<0$, we have the so-called Weibull MDA, i.e. light right-tails, with a finite right endpoint. In addition, $\gamma=0$ corresponds to the Gumbel MDA (exponential right-tails). If $\gamma>0$, we have the Fréchet MDA corresponding to heavy right-tails (polynomial tail decay, with an infinite right endpoint). Moreover, as proved in Ferreira et al. (2012), the BS CDF, $F_{T}(\cdot)$, given in (2), belongs to $\mathcal{D}_{\mathrm{M}}\left(G_{0}\right)$, and this can be heuristically inferred from Figure 1.

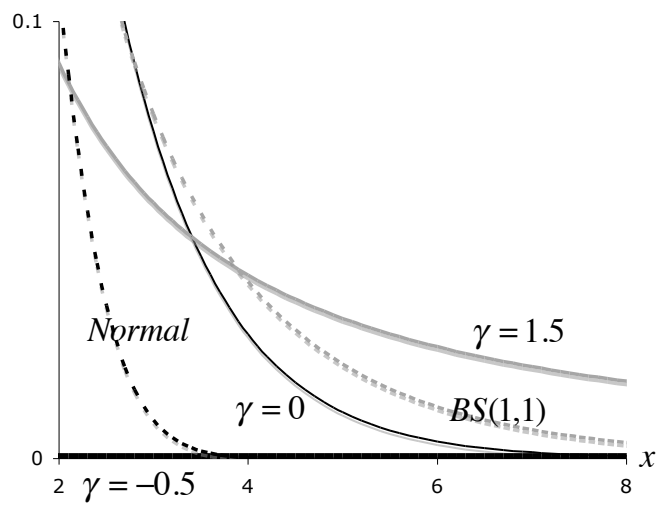

Figure 1. Right-tails of positive truncated $\mathrm{EV}_{\mathrm{M}}$ PDFs for $\gamma=-0.5, \gamma=0$ and $\gamma=1.5$, jointly with the right-tails of positive truncated normal and BS(1,1) PDFs

\section{Moments in EVBS models}

The $\mathrm{EVBS}_{\mathrm{M}}$ (and $\mathrm{EVBS}_{\mathrm{m}}$ ) distributions based on limiting EV models for maxima, $\mathrm{EV}_{\mathrm{M}}$, (and for minima, $\mathrm{EV}_{\mathrm{m}}$ ), have been introduced in Ferreira et al. (2012). Specifically, consider that the RV $Z$ follows the EV distribution for maxima given in (4), i.e. $Z \sim \operatorname{EV}_{\mathrm{M}}(\gamma)$. Then we use the notation $\operatorname{EVBS}_{\mathrm{M}}(\alpha, \delta, \gamma)$ for the RV

$$
T=\delta\left(\alpha Z / 2+\sqrt{\alpha^{2} Z^{2} / 4+1}\right)^{2} .
$$


Analogously, if we consider that $Z$ follows the EV distribution for minima given in (5), i.e. $Z \sim \operatorname{EV}_{\mathrm{m}}(\gamma)$, and the same expression for $T$, i.e. that given in (6), we use the corresponding notation $T \sim \operatorname{EVBS}_{\mathrm{m}}(\alpha, \delta, \gamma)$.

The shapes for the $\mathrm{EVBS}_{\mathrm{M}}$ and the $\mathrm{EVBS}_{\mathrm{m}} \mathrm{PDFs}$ are quite diverse. As expected, the parameter $\alpha$ can modify drastically the shapes of these distributions. In the case of thise parameter $\gamma$, we detect changes in the kurtosis and tail heaviness, as is also expected. The EVBS models are thus very flexible and with extremely diversified left and right-tails; see Ferreira et al. (2012).

The following result comes directly from Theorem 2.6 of Vilca and Leiva (2006), which allows us to state the moments of the RV $T$ given in (6).

Theorem 1. Let the $R V T$ be as given in (6). Then the rth moment of $T$ exists if $\mathrm{E}\left[Z^{k+l}\left(\{\alpha Z\}^{2}+4\right)^{(k-l) / 2}\right]<\infty$, with $k=0, \ldots, r$ and $l=0, \ldots, k$, and we have

$$
\mathrm{E}\left[T^{r}\right]=\delta^{r} \sum_{k=0}^{r}\left(\begin{array}{l}
r \\
k
\end{array}\right) \sum_{l=0}^{k}\left(\begin{array}{l}
k \\
l
\end{array}\right) 2^{k} \mathrm{E}\left[\left(\frac{\alpha Z}{2}\right)^{k+l}\left(\left\{\frac{\alpha Z}{2}\right\}^{2}+1\right)^{(k-l) / 2}\right]
$$

Particular moments of $T$ that are of interest correspond to the mean, $\mu[T]=\mathrm{E}[T]$, the variance, $\sigma^{2}[T]=\mathrm{V}[T]$, the standard deviation (SD), $\sigma[T]=\sqrt{\mathrm{V}[T]}$, and the coefficients of variation $(\mathrm{CV}), \delta[T]=\sigma[T] / \mu[T]$, of skewness (CS), $\beta_{1}[T]=\mathrm{E}\left[(\{T-\mu[T]\} / \sigma[T])^{3}\right]$ and of kurtosis $(\mathrm{CK})$, $\beta_{2}[T]=\mathrm{E}\left[(\{T-\mu[T]\} / \sigma[T])^{4}\right]$, as well as the excess kurtosis (EK), $\alpha_{4}[T]=$ $\delta_{2}[T]-3$.

In order to obtain these moments for the $\mathrm{EVBS}_{\mathrm{M}}$ and $\mathrm{EVBS}_{\mathrm{m}} \mathrm{RV}$, we need to have information on the moments of the $\mathrm{EV}_{\mathrm{M}}$ and $\mathrm{EV}_{\mathrm{m}}$ models given in (4) and (5) respectively, which will be sketched next.

For an RV, $X_{\mathrm{M}}$, with an $\operatorname{EV}_{\mathrm{M}}(\gamma)$ distribution, we have

$$
\begin{aligned}
& \mu\left[X_{\mathrm{M}}\right]= \begin{cases}\{\Gamma(1-\gamma)-1\} / \gamma, & \text { if } \gamma<1(\neq 0), \\
-\psi(1), & \text { if } \gamma=0,\end{cases} \\
& \sigma^{2}\left[X_{\mathrm{M}}\right]= \begin{cases}\left(l_{2}-l_{1}^{2}\right) / \gamma^{2}, & \text { if } \gamma<1 / 2(\neq 0), \\
\pi^{2} / 6, & \text { if } \gamma=0,\end{cases}
\end{aligned}
$$


where $l_{k}=\Gamma(1-k \gamma)$, for $k=1, \ldots, 4$, and $-\psi(1)$ is the Euler constant, with $\psi=\Gamma^{\prime} / \Gamma$ being the digamma function, i.e. the logarithmic derivative of the gamma function, denoted as usual by $\Gamma$, with $\Gamma^{\prime}$ being its derivative. In addition,

$$
\begin{aligned}
& \beta_{1}\left[X_{\mathrm{M}}\right]= \begin{cases}\left(l_{3}-3 l_{1} l_{2}+2 l_{1}^{3}\right) /\left(l_{2}-l_{1}^{2}\right)^{3 / 2}, & \text { if } \gamma<1 / 3(\neq 0), \\
12 \sqrt{6} \zeta(3) / \pi^{3}, & \text { if } \gamma=0,\end{cases} \\
& \alpha_{4}\left[X_{\mathrm{M}}\right]= \begin{cases}\left\{l_{4}-4 l_{1} l_{3}+6 l_{1}^{2} l_{2}-3 l_{1}^{4}\right\} /\left(l_{2}-l_{1}^{2}\right)^{2}, & \text { if } \gamma<1 / 4(\neq 0), \\
27 / 5, & \text { if } \gamma=0,\end{cases}
\end{aligned}
$$

where

$$
\zeta(k)=\sum_{j=0}^{\infty} j^{-k}
$$

is the Riemann zeta function, for $k>1$.

Similarly, given the relation $G_{\gamma}^{*}(x)=1-G_{\gamma}(-x)$ mentioned in Remark 1, if we consider $X_{\mathrm{m}}$, with an $\operatorname{EV}_{\mathrm{m}}(\gamma)$ distribution, we have

$$
\mathrm{E}\left[X_{\mathrm{m}}^{k}\right]=(-1)^{k} \mathrm{E}\left[X_{\mathrm{M}}^{k}\right] \quad \text { for all } k \geq 1 .
$$

Values of the mean, SD, CS and EK for $\mathrm{EVBS}_{\mathrm{M}}$ and $\mathrm{EVBS}_{\mathrm{m}}$ distributions can be found in Table 1.

Example. The Pareto distribution is very common in the domain of heavytailed models, i.e. models in the Fréchet MDA, necessarily with $\gamma>0$. Let $Z \sim \operatorname{Pareto}(\gamma)$, i.e., $F_{Z}(x)=1-x^{-1 / \gamma}$, with $x \geq 1$. Then, for $s$ non-null and if $\gamma<1 /(s+1)$,

$$
\mathrm{E}\left[Z^{s} \sqrt{(\alpha Z)^{2}+4}\right]=\frac{\alpha_{2} F_{1}\left(-\frac{1}{2},-\frac{1}{2}\left(s+1-\frac{1}{\gamma}\right), \frac{1-\gamma(s+1)}{2 \gamma},-\frac{4}{\alpha^{2}}\right)}{1-\gamma(s+1)},
$$

where ${ }_{2} F_{1}$ is the hypergeometric function (see Abramowitz and Stegun, 1972) given by

$$
{ }_{2} F_{1}(a, b, c, x)=\sum_{k=0}^{\infty} \frac{a(a+1) \cdots(a+k-1) b(b+1) \cdots(b+k-1)}{c(c+1) \cdots(c+k-1)} \frac{x^{k}}{k !} .
$$

The computations of the moments given in (7) of an associated BST RV then become then much simpler. 
Table 1. Values of mean, SD, CS and EK for the indicated distributions when $\delta=1$

\begin{tabular}{|c|c|c|c|c|c|c|c|c|c|}
\hline \multirow[b]{2}{*}{$\gamma$} & \multirow[b]{2}{*}{$\alpha$} & \multirow[b]{2}{*}{$\mu[T]$} & \multicolumn{3}{|c|}{$\mathrm{EVBS}_{\mathrm{m}}$ distribution } & \multicolumn{4}{|c|}{$\mathrm{EVBS}_{\mathrm{M}}$ distribution } \\
\hline & & & $\sigma[T]$ & $\beta_{1}[T]$ & $\alpha_{4}[T]$ & $\mu[T]$ & $\sigma[T]$ & $\beta_{1}[T]$ & $\alpha_{4}[T]$ \\
\hline \multirow[t]{5}{*}{1.50} & 0.05 & 0.850 & 0.272 & -1.964 & 2.789 & - & - & - & - \\
\hline & 0.10 & 0.800 & 0.320 & -1.385 & 0.610 & - & - & - & - \\
\hline & 0.50 & 0.693 & 0.472 & -0.217 & -1.471 & - & - & - & - \\
\hline & 1.00 & 0.709 & 0.618 & 0.288 & -1.418 & - & - & - & - \\
\hline & 1.50 & 0.776 & 0.787 & 0.599 & -1.101 & - & - & - & - \\
\hline \multirow[t]{5}{*}{1.00} & 0.05 & 0.898 & 0.205 & -2.535 & 6.419 & - & - & - & - \\
\hline & 0.10 & 0.850 & 0.263 & -1.696 & 2.115 & - & - & - & - \\
\hline & 0.50 & 0.740 & 0.463 & -0.183 & -1.294 & - & - & - & - \\
\hline & 1.00 & 0.767 & 0.658 & 0.449 & -1.094 & - & - & - & - \\
\hline & 1.50 & 0.860 & 0.893 & 0.841 & -0.501 & - & - & - & - \\
\hline \multirow[t]{5}{*}{0.50} & 0.05 & 0.943 & 0.122 & -2.927 & 11.913 & - & - & - & - \\
\hline & 0.10 & 0.905 & 0.186 & -1.842 & 3.942 & - & - & - & - \\
\hline & 0.50 & 0.804 & 0.452 & 0.003 & -0.983 & - & - & - & - \\
\hline & 1.00 & 0.852 & 0.735 & 0.803 & -0.213 & - & - & - & - \\
\hline & 1.50 & 0.989 & 1.096 & 1.311 & 1.099 & - & - & - & - \\
\hline \multirow[t]{5}{*}{0.25} & 0.05 & 0.961 & 0.084 & -2.177 & 8.277 & 1.052 & - & - & - \\
\hline & 0.10 & 0.930 & 0.145 & -1.459 & 3.074 & 1.121 & - & - & - \\
\hline & 0.50 & 0.843 & 0.449 & 0.233 & -0.651 & 2.443 & - & - & - \\
\hline & 1.00 & 0.910 & 0.803 & 1.126 & 0.872 & 6.236 & - & - & - \\
\hline & 1.50 & 1.083 & 1.272 & 1.706 & 2.953 & 12.496 & - & - & - \\
\hline \multirow[t]{5}{*}{0.00} & 0.05 & 0.974 & 0.060 & -0.863 & 1.242 & 1.031 & 0.069 & 1.461 & 4.219 \\
\hline & 0.10 & 0.952 & 0.114 & -0.624 & 0.519 & 1068 & 0.148 & 1.828 & 6.937 \\
\hline & 0.50 & 0.889 & 0.453 & 0.630 & 0.125 & 1.606 & 1.370 & 4.652 & 45.629 \\
\hline & 1.00 & 0.985 & 0.911 & & & 2.994 & 4.766 & 5.920 & 69.648 \\
\hline & 1.50 & 1.208 & 1.546 & 2.285 & 6.588 & 5.243 & 10.380 & 6.299 & 77.338 \\
\hline \multirow[t]{5}{*}{-0.25} & 0.05 & 0.983 & 0.050 & 0.046 & -0.249 & 1.020 & 0.052 & 0.219 & -0.204 \\
\hline & 0.10 & 0.968 & 0.098 & 0.181 & -0.192 & 1.044 & 0.107 & 0.349 & -0.106 \\
\hline & 0.50 & 0.941 & 0.476 & 1.257 & 2.230 & 1.353 & 0.693 & 1.260 & 1.864 \\
\hline & 1.00 & 1.081 & 1.092 & 2.402 & 8.4 & 2003 & 1.981 & 1.935 & 4.586 \\
\hline & 1.50 & 1.379 & 1.993 & 3.146 & 14.199 & 3.264 & 4.017 & 2.248 & 6.150 \\
\hline \multirow[t]{5}{*}{-0.50} & 0.05 & 0.990 & 0.047 & 0763 & 0,501 & 1012 & 0.046 & -0.506 & -0.028 \\
\hline & 0.10 & 0.982 & 0.094 & 0.903 & 1.020 & 1.027 & 0.093 & -0.389 & -0.238 \\
\hline & 0.50 & 0.998 & 0.538 & אי 2 & 8156 & 1200 & 0.501 & 0.338 & -0.607 \\
\hline & 1.00 & 1.211 & 1.412 & 3.629 & 21.511 & 1.699 & 1.223 & 0.877 & 0.032 \\
\hline & 1.50 & 1.623 & 2.760 & & & 2.425 & 2.284 & 1.173 & 0.663 \\
\hline \multirow[t]{5}{*}{-1.00} & 0.05 & 1.001 & 0.053 & 2.331 & 8.969 & 1.001 & 0.048 & -1.728 & 4.029 \\
\hline & 0.10 & 1.005 & 0.112 & & 13.369 & & 0.092 & -1.505 & 2.695 \\
\hline & 0.50 & 1.151 & 0.940 & 6.519 & 89.537 & 1.100 & 0.383 & -0.546 & -0.664 \\
\hline & 1.00 & 1.654 & 3.144 & 8.746 & 152.367 & 1.346 & 0.755 & -0.028 & -1.235 \\
\hline & 1.50 & 2.535 & 6.760 & 9.507 & 175.900 & 1.715 & 1.223 & 0.258 & -1.252 \\
\hline \multirow{5}{*}{-1.50} & 0.05 & 1.014 & 0.080 & 5.598 & 73.340 & 0.991 & 0.060 & -2.888 & 11.851 \\
\hline & 0.10 & 1.033 & 0.190 & 8.346 & 200.962 & 0.986 & 0.109 & -2.366 & 7.009 \\
\hline & 0.50 & 1.456 & 2.706 & 20.475 & 1130.34 & 1.027 & 0.358 & -0.988 & -0.069 \\
\hline & 1.00 & 2.749 & 10.369 & 22.496 & 1317 & 1.181 & 0.610 & -0.458 & -1.157 \\
\hline & 1.50 & 4.929 & 23.121 & 22.958 & 1361.20 & 1.413 & 0.893 & -0.180 & -1.435 \\
\hline
\end{tabular}




\section{Estimation and validation in EVBS models}

Estimation aspects and model checking for EVBS distributions have been dealt with in Ferreira et al. (2012). The system of likelihood equations does not produce an explicit solution, and so a numerical procedure is necessary. An $\mathrm{R}$ package named evbs, to analyse data from EVBS models, is being developed, and its "in progress" version is already available through the authors. This package contains diverse indicators, as well as methodologies useful for EVBS distributions, for example maximum likelihood (ML) estimation of the unknown parameters of the distribution.

Once the EVBS distribution parameters have been estimated, a natural question that arises is that of checking how good is the fit of the model to the data. In order to compare the EVBS distributions with other distributions, we can use model selection criteria based on loss of information, such as Akaike (AIC) and Schwarz's Bayesian (BIC) information criteria. These criteria are given by

$$
\mathrm{AIC}=-2 \ell(\widehat{\boldsymbol{\theta}})+2 d \quad \text { and } \quad \mathrm{BIC}=-2 \ell(\widehat{\boldsymbol{\theta}})+d \log (n),
$$

where $\ell(\boldsymbol{\theta})$ is the $\log$-likelihood function for the parameter $\boldsymbol{\theta}$ associated

with the model, $\widehat{\boldsymbol{\theta}}$ is its ML estimate, $n$ is the sample size and $d$ is the dimension of the parameter space.

Remark 2. AIC and BIC are based on a penalization of the likelihood function that allows us to compare models with different numbers of parameters, because, as is known, models with more parameters always provide a better fit. Thus a model whose $\mathrm{AIC}$ or $\mathrm{BIC}$ has the smallest value is better; see Sanhueza et al. (2008) and Ferreira et al. (2012). This is an important point, because the EVBS distribution has more parameters than its closer competitors, such as the $\mathrm{BS}$ and $\mathrm{EV}$ distributions.

Because, in general, differences between two values of the BIC are not very noticeable, the Bayes factor $(\mathrm{BF})$ can be used to highlight such differences, if they exist. An interpretation of a transformation of the $\mathrm{BF}\left(B_{12}\right)$, denoted by $2 \log \left(B_{12}\right)$, which allows us to detect the degree of superiority of one model (Model 1) with respect to another (Model 2), is given in Table 2. For details, see Ferreira et al. (2012) and references therein. 
Table 2. Interpretation of $2 \log \left(B_{12}\right)$ associated with the $\mathrm{BF}$

\begin{tabular}{cc}
\hline $2 \log \left(B_{12}\right)$ & Evidence in favour of $\mathrm{M}_{1}$ \\
\hline$<0$ & Negative $\left(\mathrm{M}_{2}\right.$ is accepted $)$ \\
{$[0,2)$} & Weak \\
{$[2,6)$} & Positive \\
{$[6,10)$} & Strong \\
$\geq 10$ & Very strong \\
\hline
\end{tabular}

\section{Hazard analysis in EVBS models}

A hazard may be considered as a dangerous event that can lead to an emergency or disaster. A hazard analysis can be performed statistically based on the hazard rate (HR), also known as chance function, failure rate, intensity function, or risk rate, among other names. A nice property of the HR is that it allows us to better characterize the behaviour of statistical distributions, and to differentiate models with very similar CDFs. For example, the HR may have several different shapes, such as increasing (IHR), constant (exponential distribution), decreasing (DHR), bathtub (BT), inverse bathtub (IBT) approaching a non-null constant, or IBT approaching zero. The HR of $T$ is given in general by

$$
h_{T}(t):=\frac{f_{T}(t)}{1-F_{T}(t)}, \quad t>0, \quad 0<F_{T}(t)<1,
$$

where $f_{T}$ and $F_{T}$ are the PDF and the CDF of $T$.

A simple manner for exploring the shape of the HR of a RV $T$ is by its corresponding scaled total time on test (TTT) function given by

$$
W_{T}(u)=\int_{0}^{F_{T}^{-1}(u)}\left(1-F_{T}(y)\right) d y / H_{T}^{-1}(1), \quad 0 \leq u \leq 1,
$$

which can be empirically approximated, making it possible to construct the empirical scaled TTT curve by plotting the consecutive points $\left[k / n, W_{n}(k / n)\right]$, where $W_{n}(k / n)=\left\{\sum_{i=1}^{k} T_{i: n}+(n-k) T_{k: n}\right\} / \sum_{i=1}^{n} T_{i: n}$, for $k=1, \ldots, n$, with $T_{i: n}$ being the corresponding $i$ th ascending order statistic, for $1 \leq i \leq n$, associated with the observed sample $\left(T_{1}, T_{2}, \ldots, T_{n}\right)$. From Figure 2, we identify several theoretical shapes for the scaled TTT curve. Thus, a TTT curve that is concave (or convex) is related to the IHR (or DHR) class. A concave (or convex) and then convex (or concave) TTT curve is related to a BT (or IBT) HR. Finally, a TTT curve expressed by a straight line corresponds to the exponential distribution. 


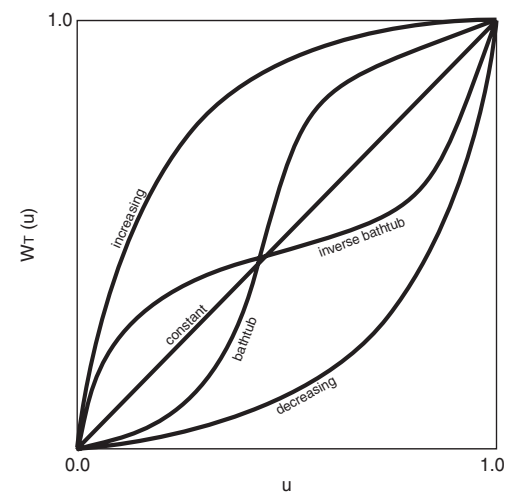

Figure 2. Theoretical scaled TTT curves for a general model with the indicated HR shape

The $\mathrm{EVBS}_{\mathrm{M}}$ and $\mathrm{EVBS}_{\mathrm{m}}$ distributions provide very rich models, in the sense that they can attain all types of TTT curves given above. For more details about hazard analysis for EVBS models, see Ferreira et al. (2012).

\section{An application to biometry}

For illustration purposes, we consider the uncensored part of a data set analysed by Leiva et al. (2007) corresponding to the survival times ( $T$, in months) of 48 patients who were treated with alkylating agents for multiple myeloma. These data (which we will henceforth call myeloma) are: 1, 1, $2,2,2,3,5,5,6,6,6,6,7,7,7,9,11,11,11,11,11,13,14,15,16,16,17$, $17,18,19,19,24,25,26,32,35,37,41,42,51,52,54,58,66,67,88,89$, 92.

Table 3. Descriptive statistics for myeloma data (in months)

\begin{tabular}{cccccccccc}
\hline Median & Mean & SD & CV & CS & CK & Range & Min. & Max. & $n$ \\
15.500 & 24.44 & 24.67 & $100 \%$ & 1.36 & 6.22 & 18 & 1 & 92 & 48 \\
\hline
\end{tabular}

For analysing the myeloma data, we use the implementation in $\mathrm{R}$ code of the EVBS models considered in Ferreira et al. (2012). An exploratory data analysis (EDA) of such data is first produced, and then estimation and EVBS model checking are carried out. The EDA of myeloma, based on the descriptive summary presented in Table 3 and in Figure 3, allows us to detect a positively skewed distribution with a moderate to high kurtosis and a shape for the HR, all of which can be modelled well by an EVBS distribution. Thus we propose this distribution for describing the myeloma data. 

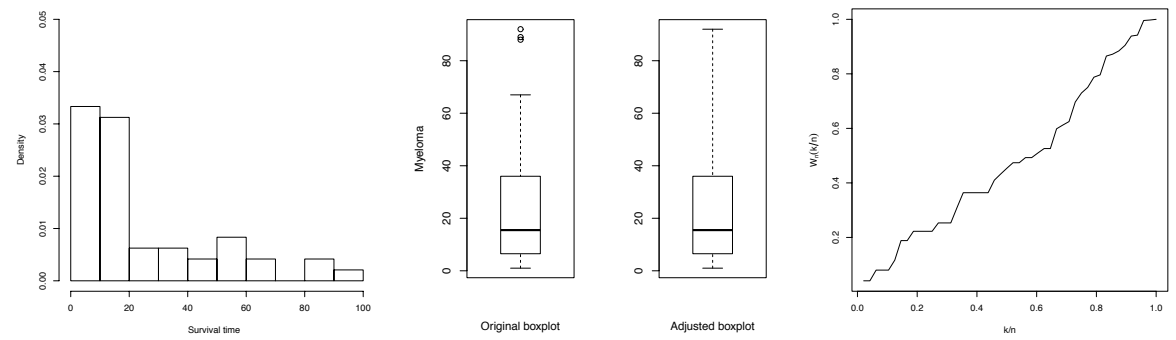

Figure 3. Histogram (left) and indicated boxplots (centre) and TTT plot

(right) for myeloma

As the $\mathrm{EVBS}_{\mathrm{m}}$ distribution based on the Gumbel min $_{\text {model, }} \Lambda^{*}(x)=$ $G_{0}^{*}(x)$, given in (5), belongs to the Gumbel min-domain of attraction, we apply a semi-parametric EV test to analyse whether or not the myeloma data belongs to this domain. We test $\mathrm{H}_{0}: F \in \mathcal{D}_{\mathrm{m}}\left(G_{\gamma}^{*}\right)$, with $\gamma \geq 0$, against $\mathrm{H}_{1}: F \notin \mathcal{D}_{\mathrm{m}}\left(G_{\gamma}^{*}\right)$, with $\gamma \geq 0$. From Figure 4 , we see the sample path of the test statistic as a function of the $k$ largest order statistics and the critical value (horizontal line) above which we reject $\mathrm{H}_{0}$. For myeloma, we do not reject the null hypothesis for $1 \leq k \leq 36$, which is a credible result in EVT to keep such a hypothesis. Note also that we cannot have $\gamma>0$ due to the fact that a infinite left endpoint does not make sense for these data. We have just restricted to the $\operatorname{EVBS}_{\mathrm{m}}(\alpha, \delta) \equiv \operatorname{EVBS}_{\mathrm{m}}(\alpha, \delta, 0)$ model.

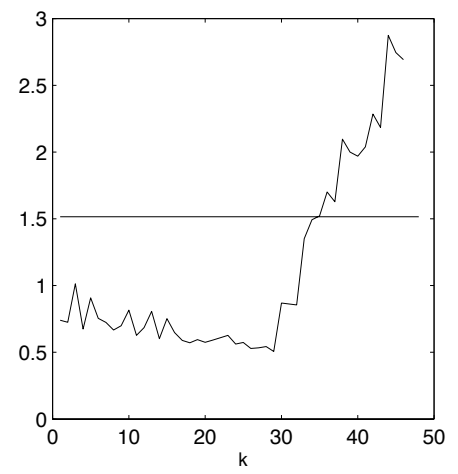

Figure 4. Sample path of the EV condition test applied to myeloma (horizontal line: critical value above which we reject $F \in \mathcal{D}_{\mathrm{m}}\left(G_{\gamma}^{*}\right)$, with $\gamma \geq 0$ )

Once we have established the type of EVBS model to be used, we estimate the model parameters using the evbs package and myeloma data. These results, along with the negative value of the log-likelihood function and values for $\mathrm{AIC}, \mathrm{BIC}$ and $\mathrm{BF}$, are given in Table 4 . 
Table 4. ML estimates, AIC, BIC and BF for the indicated models using myeloma

\begin{tabular}{|c|c|c|c|c|c|c|c|}
\hline Distribution & $\widehat{\theta}_{1}$ & $\widehat{\theta}_{2}$ & $\widehat{\theta}_{3}$ & $-\ell$ & $\mathrm{AIC}$ & $\mathrm{BIC}$ & $2 \log \left(B_{1}\right.$ \\
\hline$\overline{\operatorname{EVBS}_{\mathrm{m}}(\alpha, \delta)}$ & 1.115 & 23.684 & - & 199.981 & 403.962 & 409.469 & - \\
\hline$\sigma, \gamma)$ & 10.182 & 10.2 & 0.61 & & & 420 & 520 \\
\hline $\mathrm{GP}($ & - & 24.467 & -0.001 & 201 & 40 & 412 & 2.866 \\
\hline $3 \mathrm{~S}(\alpha, \delta)$ & 1.323 & 12.719 & - & 201.494 & 406.988 & 412.495 & 3.026 \\
\hline
\end{tabular}

We perform a comparison among the $\operatorname{EVBS}_{\mathrm{m}}$ and $\mathrm{BS}, \mathrm{EV}_{\mathrm{M}}(\mu, \sigma, \gamma):=$ $\mu+\sigma \operatorname{EV}_{\mathrm{M}}(\gamma)$ and generalized Pareto (GP) models using these criteria. Note that the $\operatorname{GP}(\sigma, \gamma) \mathrm{CDF}$, another important model in EVT, is related to the GEV CDF, in (4), through $P_{\gamma}(x ; \sigma)=1+\ln G_{\gamma}(x / \sigma), 1+\gamma x / \sigma>0, x>0$. This comparison indicates that the $\mathrm{EVBS}_{\mathrm{m}}$ model is strongly superior to the GEV model for these data, and with positive evidence in its favour in relation to the BS and GP models. The excellent agreement between the $\mathrm{EVBS}_{\mathrm{m}}$ distribution and the myeloma data can be observed in Figure 5 from the histogram of the data with estimated $\mathrm{EVBS}_{\mathrm{m}} \mathrm{PDF}$ (left), the empirical CDF plot with estimated $\mathrm{EVBS}_{\mathrm{m}} \mathrm{CDF}$ (centre) and the QQ plot (right).

Based on this analysis, we can use the $\mathrm{EVBS}_{\mathrm{m}}$ model for obtaining different indicators useful in survival analysis, such as the hazard rate and survival function, in order to predict times of survival and evaluate changes in treatment dose.
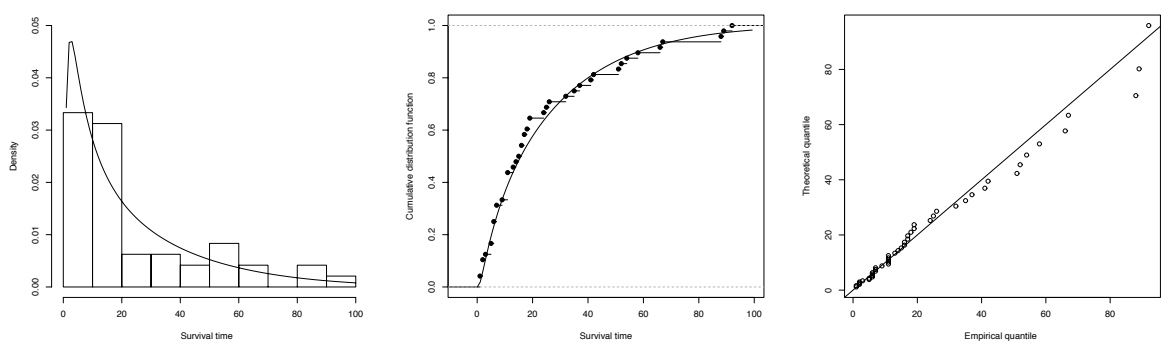

Figure 5. Histogram with estimated $\mathrm{EVBS}_{\mathrm{m}}\left(\mathrm{Gumbel}_{\mathrm{min}}\right) \mathrm{PDF}$ (left), empirical $\mathrm{CDF}$ plot with estimated (theoretical) $\mathrm{EVBS}_{\mathrm{m}} \mathrm{CDF}$ (center) and QQ plot

(right) for myeloma 


\section{Concluding remarks}

We have dealt with extreme value versions of the Birnbaum-Saunders distribution, which were introduced by Ferreira et al. (2012). A description of the moments and hazard analysis of extreme value Birnbaum-Saunders distributions has been given. We have used the $\mathrm{R}$ package initiated in Ferreira et al. (2012) for analysing a real data set corresponding to survival times of patients who were treated with alkylating agents for multiple myeloma. This analysis has allowed us to show the adequacy of these new statistical distributions, in a pure parametric framework, and identify them as models that can be useful for diverse medical practitioners in order predict times of survival for such patients and evaluate changes in their treatment dose.

\section{Acknowledgments}

Marta Ferreira was partially supported by the Research Centre of Mathematics of the University of Minho through the FCT Pluriannual Funding Program and by PTDC/FEDER grants from Portugal. M. Ivette Gomes was partially supported by national funds through FCT (Fundação para a Ciência e a Tecnologia) by PEst-OE/MAT/UI0006/2011, FCT/OE, POCI 2010 and PTDC/FEDER grants from Portugal. Víctor Leiva was supported by FONDECYT (Fondo Nacional de Desarrollo Cientíco y Tecnológico) by grant 1080326 from Chile.

\section{REFERENCES}

Abramowitz M., Stegun I.A. (1972): Handbook of Mathematical Functions with Formulas, Graphs, and Mathematical Tables. Dover, New York.

Birnbaum Z.W., Saunders S.C. (1969): A new family of life distributions. J. Applied Probab. 6: 319-327.

Ferreira M., Gomes M.I., Leiva V. (2012): On an Extreme Value Version of the Birnbaum-Saunders Distribution. Revstat (in press).

Fréchet M. (1927): Sur la loi de probabilité de l'écart maximum. Ann. Société Polonaise de Mathématique 6: 93-116.

Fisher R.A., Tippett L.H.C. (1928): Limiting forms of the frequency of the largest or smallest member of a sample. Proc. Cambridge Phil. Soc. 24: 180-190.

Gnedenko B.V. (1943): Sur la distribution limite du terme maximum d'une série aléatoire. Ann. Math. 44: 423-453.

Gomes M.I., Canto e Castro L., Fraga Alves M.I., Pestana D. (2008): Statistics of extremes for iid data and breakthroughs in the estimation of the extreme value index: Laurens de Haan leading contributions. Extremes 11(1): 3-34. 
Jenkinson A.F. (1955): The frequency distribution of the annual maximum (or minimum) values of meteorological elements. Quart. J. Royal Meteorol. Society 81: $158-171$.

Leiva V., Barros M., Paula G.A., Galea, M. (2007): Influence diagnostics in logBirnbaum-Saunders regression models with censored data. Comp. Stat. Data Anal. 51: 5694-5707.

Sanhueza A., Leiva V., Balakrishnan N. (2008): The generalized BirnbaumSaunders distribution and its theory, methodology and application. Comm. Statist. - Theory and Methods 37: 645-670.

Mises R. von (1936): La distribution de la plus grande de $n$ valeurs. Revue Math. Union Interbalcanique, 1, 141-160. Reprinted in Selected Papers of Richard von Mises. Amer. Math. Soc. 2(1964): 271-294.

Vilca F., Leiva V. (2006): A new fatigue life model based on the family of skewelliptical distributions. Comm. Statist. - Theory and Methods 35: 229-244. 